\title{
Mycosis fungoides mimicking tinea pedis
}

\author{
Steven A. Hanna BSc, Mark G. Kirchhof MD PhD
}

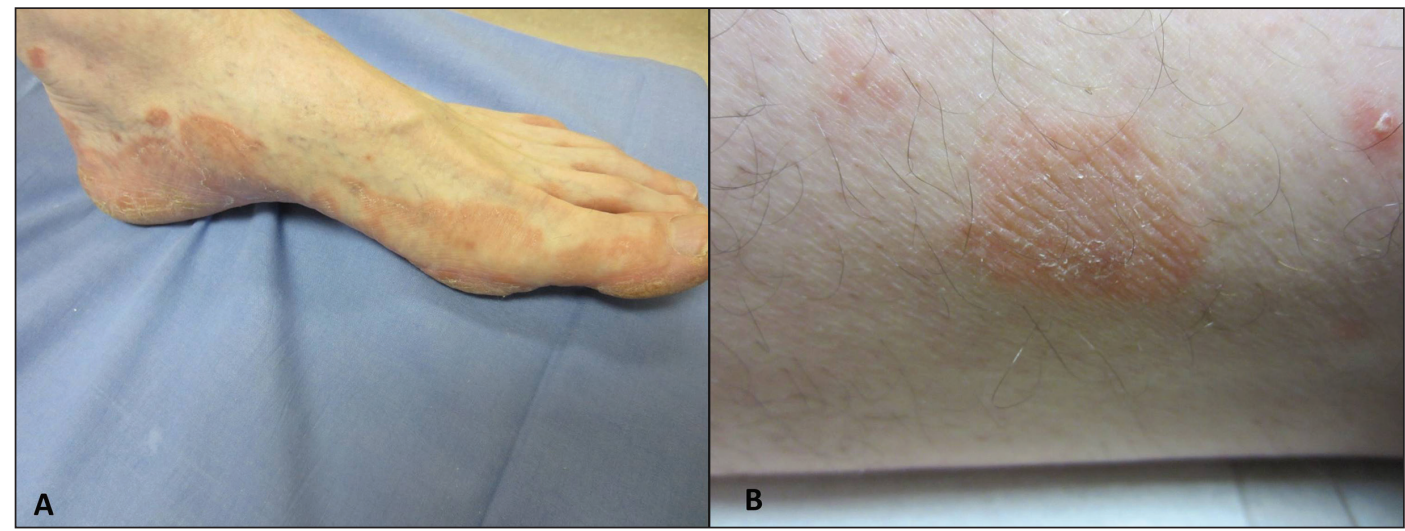

Figure 1: Mycosis fungoides resembling tinea pedis in a 68-year-old man. A) View of the left foot showing polycyclic erythematous scaly papules and plaques that mimick tinea pedis. B) View of the right upper thigh showing an erythematous plaque with fine scales and "cigarette paper" wrinkling.

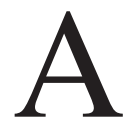
68-year-old man presented to primary care with a rash consisting of polycyclic (overlapping circles) erythematous scaly papules and plaques with raised borders (Figure 1A) that was localized to his feet. The patient was treated appropriately for tinea pedis with topical terbinafine. After several follow-up visits, the lesions showed no improvement, and the patient was prescribed oral terbinafine, in accordance with current treatment guidelines. ${ }^{1}$ After one year of treatment, there was little effect, and the patient was referred to the dermatology service.

When the patient was assessed in the dermatology clinic three months later, the rash had progressed to red-brown papules and plaques scattered over the body, including areas not exposed to the sun. These lesions had associated fine scales and "cigarette paper" wrinkling (Figure 1B). A biopsy of a lesion on the right thigh showed features suggestive of mycosis fungoides (Appendix 1, available at www.cmaj.ca/lookup/suppl/ doi:10.1503/cmaj.160038/-/DC1). Based on the biopsy results and our clinical examination, we diagnosed mycosis fungoides. We prescribed topical clobetasol ointment and betamethasone valerate $(0.1 \%)$ ointment, and administered narrowband ultraviolet B phototherapy three times per week. After three months, the lesions were almost completely cleared, including those on the feet.
The patient continued maintenance phototherapy treatment and has remained nearly free of lesions.

Mycosis fungoides, a malignant disease of $\mathrm{CD}^{+} \mathrm{T}$ cells, is the most common cutaneous lymphoma, with an incidence of about 6.4-9.6 cases per million per year in the United States. ${ }^{2}$ Treatment options include topical corticosteroids, narrow-band ultraviolet B phototherapy, topical nitrogen mustard and topical retinoids. Patients with early-stage disease have similar life expectancies to controls matched by age, sex and race. ${ }^{3}$

Clinical diagnosis of mycosis fungoides is difficult because, similar to other cutaneous lymphomas, ${ }^{4}$ it may mimic numerous cutaneous conditions. These include eczema, folliculitis, contact dermatitis and psoriasis.

Where lesions are refractory to standard therapy and areas not exposed to the sun are involved, prompt referral for biopsy, diagnosis and treatment is prudent.

\section{References}

1. Gupta AK, Cooper EA. Update in antifungal therapy of dermatophytosis. Mycopathologia 2008;166:353-67.

2. Jawed SI, Myskowski PL, Horwitz S, et al. Primary cutaneous T-cell lymphoma (mycosis fungoides and Sézary syndrome): part I. Diagnosis: clinical and histopathologic features and new molecular and biologic markers. J Am Acad Dermatol 2014;70:205.e1-e16.

3. Jawed SI, Myskowski PL, Horwitz S, et al. Primary cutaneous T-cell lymphoma (mycosis fungoides and Sézary syndrome): part II. Prognosis, management, and future directions. J Am Acad Dermatol 2014;70:223.e1-e17.

4. Wang SC, Mistry N. Woringer-Kolopp disease mimicking psoriasis. CMAJ 2015;187:1310.
Competing interests: None declared.

This article has been peer reviewed.

The authors have obtained patient consent.

Affiliations: School of Medicine (Hanna), Faculty of Health Sciences; Division of Dermatology, Department of Medicine (Kirchhof), Queen's University, Kingston, Ont.

Correspondence to: Mark Kirchhof, kirchhof.mark@gmail.com

CMAJ 2016. DOI:10.1503 /cmaj.160038 